\title{
Transanal total mesorectal excision (TaTME) for inflammatory bowel disease (IBD): review of technique and initial experience
}

Pramodh Chandrasinghe ${ }^{1,2}$, Raimund Strouhal ${ }^{2}$, Narasimhaiah Srinivasaiah ${ }^{2}$, Cosimo Alex Leo $^{2}$, Sanjeev Samaranayake ${ }^{2}$, Janindra Warusavitarne ${ }^{2}$

${ }^{1}$ Department of Surgery, Faculty of Medicine, University of Kelaniya, Sri Lanka

${ }^{2}$ Department of Colorectal Surgery, St.Mark's Hospital, United Kingdom, HA1 3UJ.

Key words: Trans anal surgery; TaTME; inflammatory bowel disease; pouch surgery

\begin{abstract}
Introduction

Trans anal minimal invasive surgery (TAMIS) is a novel technique gaining popularity in colorectal surgery due to its precision in pelvic dissection and easy accessibility to the distal rectum. Its use in colorectal cancer surgery is well documented although inflammatory bowel disease (IBD) poses a unique set of disease-specific and procedure-related challenges. Unlike in cancer surgery, the wide disease spectrum with varying morphological changes in IBD would require the surgeon to adapt accordingly from port insertion to wound closure. This article describes our experience with the first 60 procedures.
\end{abstract}

\section{Methodology}

Patients affected by IBD requiring proctectomy with or without total colectomy from 2013 to 2016 were offered Trans anal total mesorectal excision (TaTME) on a TAMIS and Single Incision Laparoscopy (SILS) combined platform. Airseal ${ }^{\circledR}$ insufflation on GelpointPath ${ }^{\circledR}$ platform with monopolar diathermy was used for rectal surgery. A second team using ultrasonic dissection carried out concomitant abdominal dissection. Procedural modifications were adopted based on authors' personal experience. Standard ileoanal $\mathrm{S}$ pouch with stapler anastomosis was performed. Surgical time, blood loss and patient demographics were recorded.

\section{Results}

All 60 patients (male - 44; median age 42.5; range 19-75) presented during the study period underwent TaTME for the rectal dissection with an $8 \%$ conversion rate. Of the total 38 $(63 \%)$ were done for ulcerative colitis and the perineal phase

Correspondence: Pramodh Chandrasinghe

E-mail: pramodh@kln.ac.lk

Received: 22-11-2017 Accepted: 13-12-2017

(iD) http://orcid.org/0000-0002-3485-961X

DOI: http://doi.org/10.4038/sljs.v35i4.8431

The Sri Lanka Journal of Surgery 2017; 35(4): 3-7 has taken a median time of 141.8 minutes. Ileo-anal pouch surgery was performed in $27(45 \%)$ patients. Two patients $(3.3 \%)$ required re-intervention due to complications in the abdominal procedure. Two patients required vacuum dressing for wound closure.

\section{Conclusion}

TaTME is a safe and feasible procedure in IBD surgery. Specific difficulties due to the inflammatory process which results in difficult dissection can be overcome with attention to anatomical details and the use of specific instruments.

\section{Introduction}

Total mesorectal excision (TME) with trans anal minimal invasive surgery (TAMIS) was developed on the platform of transanal endoscopic microsurgery (TEM) described by Buess and colleagues [1]. It is believed that quality of the rectal cancer specimens can be improved and the challenges faced during deep pelvic dissection can be overcome through this 'bottom-up' technique. The transanal procedure is combined with either laparoscopic or single incision laparoscopic approach from the abdomen. Atallah et al published the first series of TAMIS-TME in 20 patients with rectal cancer [2]. Although evidence on the efficacy of TAMIS-TME in rectal cancer has been accumulating [3, 4]. there is scarce data on its use in inflammatory bowel disease. Completion proctectomy due to intractable perineal disease or proctectomy with ileoanal pouch creation in suitable patients are the two most common procedures performed in this setting. Although completeness of circumferential resection margin is not mandatory in benign disease, there are unique issues pertaining to patients with inflammatory bowel disease (IBD) in the peri-operative period during rectal surgery. IBD encompasses ulcerative colitis (UC), Crohn's disease (CD) and indeterminate colitis in a small proportion of patients. Effects of chronic inflammation obscure the anatomical planes making dissection difficult and also affect the postoperative wound management. Use of specific instruments and devices can help in improving surgical outcome. In this article, we aim to describe the specific issues related to performing Transanal rectal excision (TaTME) in the setting of IBD and the initial learning experience from the first 60 cases performed at the St. Mark's Hospital, London. 
Difficult phases and methods to overcome them are described based on the authors' personal experience.

\section{Methodology}

\section{Platform}

There are many platforms used for transanal surgery such as Transanal endoscopic operation $(T E O \circledast)$ proctoscope (KarlStorz, Tuttlingen, Germany), Endorec Trocar (Aspide Medical), GelPOINTPath ${ }^{\circledR}$ Transanal Access Platform (Applied Medical, Rancho Santa Margarita, CA). We prefer the GelpointPath ${ }^{\circledR}$ port with a flexible sleeve and a gel port for port insertion [1]. This port has the advantage of increased freedom of movement [5]. GelpointPath ${ }^{\circledR}$ also provides freedom to select different port insertion sites depending on preference. The authors believe that port selection should be based on individual preference and experience.

\section{Port insertion and purse string}

The transanal phase may be performed concomitantly with the abdominal phase or in tandem either before or after. We prefer to perform the two procedures concomitantly using the single incision laparoscopy (SILS) port (Figure 2). The anal canal is accessed using a Lone Star retractor ${ }^{\circledR}$ (Lone Star Medical Products Inc., Houston, Texas, USA). For restorative procedures we use a clear anoscope to facilitate placing the purse-string suture (2/0 polypropylene) $2 \mathrm{~cm}$ above the dentate line. This allows direct visualisation of the dentate line and the exact location of the rectotomy to ensure that excess inflamed mucosa is not left behind. Dissection is initiated distal to the purse-string prior to insertion of the port under direct vision and the TME plane is entered. This step can also be performed after inserting the transanal port. For proctectomy without restoration, the purse- string is applied at the anal verge and the dissection is initiated in the interspinchteric plane.

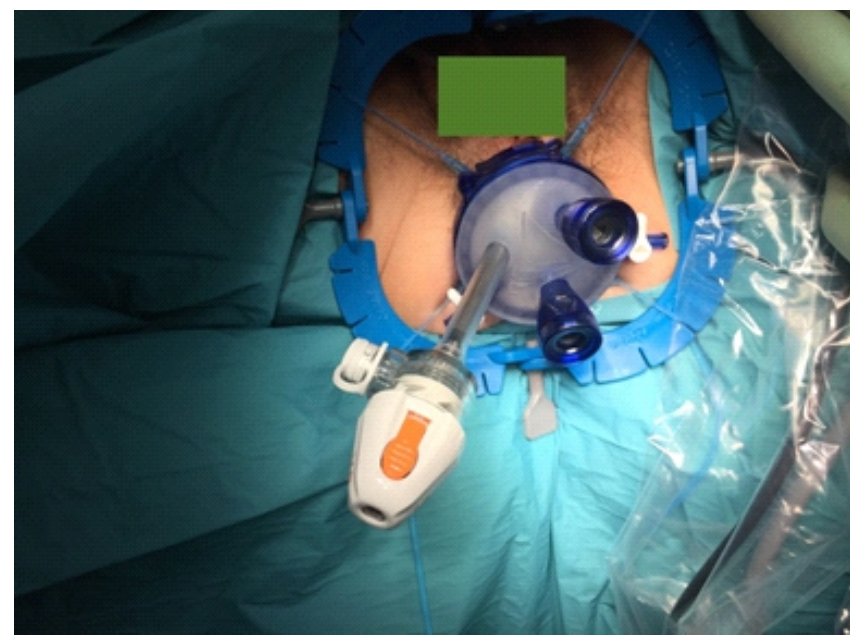

Figure 1. The arrangement of the transanal port for the trans anal dissection. The anal canal is being retracted with a 'Lonestar' retractor and the 'GelpointPath port inserted with two working ports and an 'Air-Seal' insufflation port.

\section{Insufflation}

Our initial experience was with the standard $\mathrm{CO} 2$ insufflator, although the smoke generated obscured vision. In the last 40 cases we have been using the Surgiquest Airseal ${ }^{\circledR}$ (Alder Instrument Company, Atlanta, GA, USA) device for insufflation and this provides well controlled insufflation pressure with continuous circulation preventing smoke accumulation. A specially designed Airseal ${ }^{\circledR}$ trocar is required for this and our preference is to use the $5 \mathrm{~mm}$ version (Figure 1). As the tissue planes are not well defined in the presence of inflammation, devices to reduce residual smoke and improve vision greatly facilitates dissection.

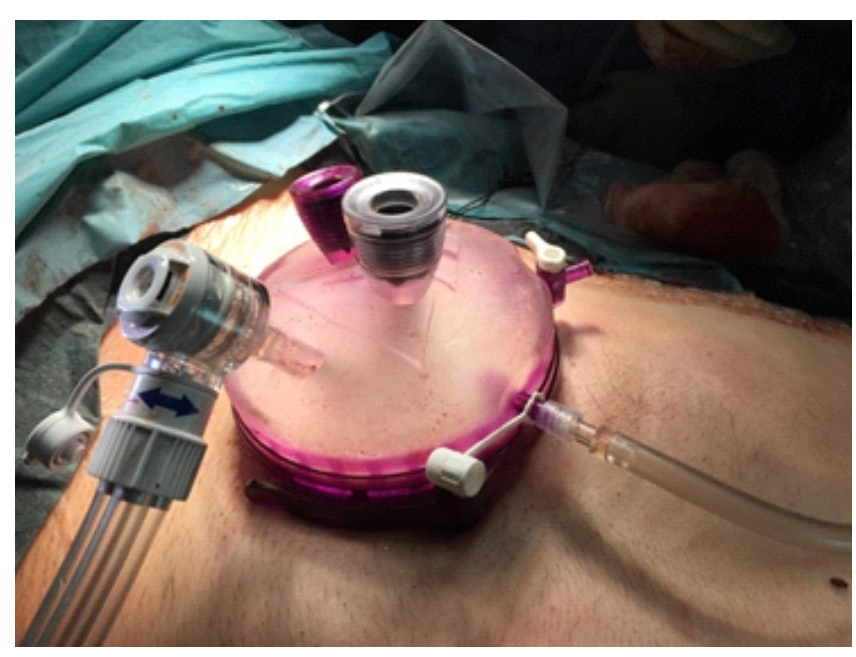

Figure 2. The arrangement for the abdominal dissection with Single Incision Laparoscopy port (SILS).

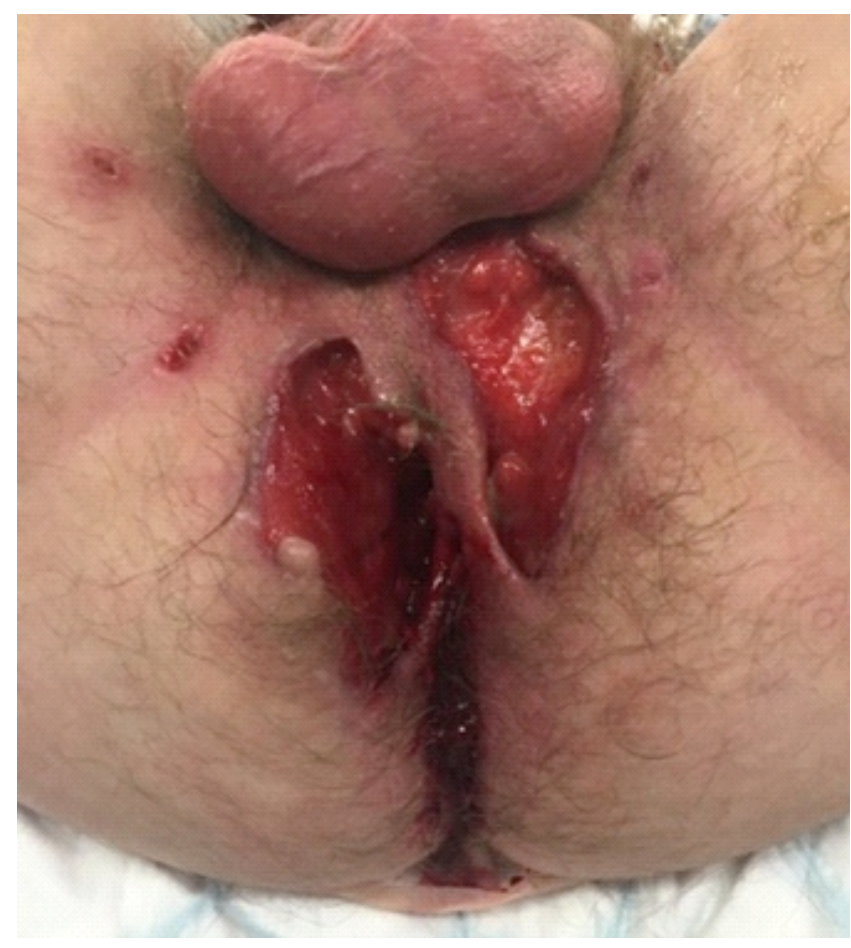

Figure 3. Severe perineal Crohn's disease with destruction of the perianal skin. 


\section{Endopelvic fascia}

The endopelvic fascia is a thickened connective tissue layer that covers the pelvic floor musculature. Once the dissection reaches the level of the anorectal junction, it is necessary to cut through the endopelvic fascia. The best site to perform this step is at 7 or $8 o^{\prime}$ clock positions. Anteriorly the pelvic floor is not well defined and posteriorly at 6'o clock the fascia forms a midline raphe, which makes dissection at these points difficult. This step is pertinent to continue the dissection in the correct plane which will otherwise tend to sway laterally and result in dissection deep to the pelvic fascia. It should be borne in mind that dissection deep to the fascia will result in troublesome bleeding.

\section{Anastomosis in restorative procedures}

The majority of UC patients undergo restorative procedures with an ileal pouch. The small bowel is extracted through the SILS port site at the RIF and the pouch formed extracorporeally. We carry out the double purse-sting technique of ileal pouch-anal anastomosis. The ileal J pouch is constructed in the standard manner using linear staplers and the anvil of the circular stapler (Chex ${ }^{\circledR}$ CS, Frankeman International, Hong Kong) secured with a No 2/0 polypropylene suture. This stapling device has a long anvil coupled with a rod and allows easier withdrawal from the anus when compared with other circular staplers. We use the Lone Star® retractor with the transparent anoscope to visualize the resection margin above the dentate. The distal purse-string is then placed transanally using a 2-0 polypropylene suture. This is not secured initially.

After completing the purse string, the pouch is guided in to the pelvis laparoscopically and the anvil is grasped with a Robert's forcep inserted through the distal purse string. Once the shaft of the anvil is pulled, through the purse-string and fixed to the spike of the circular stapler.

Subsequently the circular stapler is closed and fired after confirming the orientation of the pouch with the abdominal team. It is important to make sure the small bowel mesentery is not twisted along its axis, prior to performing the anastomosis.

\section{Perineal wound in non- restorative procedures}

The perineal wound poses a formidable challenge especially in Crohn's disease. Involvement of the perineum by fistulating disease makes it necessary to excise a significant area of perineal soft tissue, which can leave a large defect. Due to its inflamed and infected nature, primary closure is not taken up in these complicated cases. Usually we apply a vacuum dressing primarily and re-assess the wound in 4 to 6 weeks' time to decide on a flap closure with the plastic surgeons.
Table 1. Patient demographics of the first 60 cases- TaTME in IBD

\begin{tabular}{|l|l|}
\hline Cases & $\mathbf{N}=\mathbf{6 0}$ \\
\hline UC & $38(63 \%)$ \\
\hline CD & $22(37 \%)$ \\
\hline Age (median years: range) & $42.5(19-75$ \\
\hline Sex & \\
\hline \multicolumn{1}{|c|}{ Male } & $44(73 \%)$ \\
\hline Female & $16(27 \%)$ \\
\hline ASA & \\
\hline I & 14 \\
\hline II & 43 \\
\hline \multicolumn{1}{|c|}{ III } & 03 \\
\hline BMI (median: range) & $25.4(20.9-30.6)$ \\
\hline
\end{tabular}

\section{Results}

Sixty consecutive patients requiring proctectomy with or without restoration for $\mathrm{CD}$ and $\mathrm{UC}$ underwent the procedure at the unit up to July 2016. None of the patients were excluded, even in the presence of areas of perineal breakdown secondary to Crohn's disease. The median age of the patient population was 42.5 years (range 19 to 75 years) and 44 (73 $\%)$ were male. Restorative procedures with ileo-anal pouch creation were performed in 27 (45\%) of the patients (Table 1 ). Four patients $(6.7 \%)$ had colorectal cancer diagnosed in the specimen following surgery. Average surgical time taken for the transanal phase was 141.8 minutes with an $8 \%(n=5)$ conversion rate, the majority $(n=3)$ due to the dissection plane being obscured by inflammation. Two patients out of the 60 $(3.33 \%)$ required a vacuum dressing for the perineal wound management. Eight post-operative complications were experienced (Clavien-Dindo 1:4, 2:2, 3; 2). The two complications requiring re-intervention were bowel obstruction due to twisting of the mesentery and a tight ileostomy.

\section{Discussion}

Inflammatory bowel disease poses a unique series of challenges for the trans-anal approach compared to malignant disease. Severe perineal Crohn's disease may present as completely deformed anal opening (Figure 3) and with a strictured anus. However, these are not contraindications to the trans-anal approach.

The initial plane of dissection in these cases needs to be placed further away from the anal verge to encompass the diseased perineal skin and subcutaneous tissue of the perineum in the specimen. A major challenge during rectal dissection in IBD is the inflammation obscuring tissue planes (Figure 4). Although extramural inflammation is expected in $\mathrm{CD}$, our experience is that it also affects UC. In patients with long standing active UC, the TME plane is as challenging as in CD. The mesorectal fat is more friable and may bleed easily. 


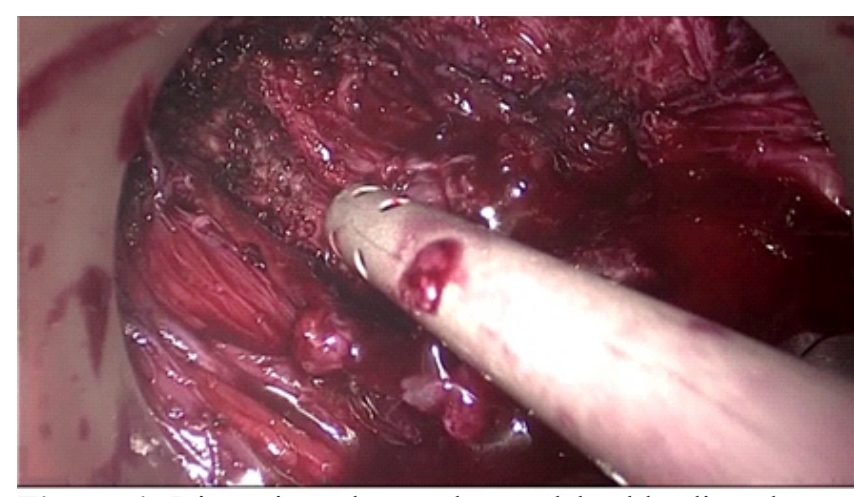

Figure 4. Dissection planes obscured by bleeding due to inflamed tissue planes. The diathermy coupled with suction device increases efficiency due to not having to change instruments.

The ENDOPATH Electrosurgery Probe Plus ${ }^{\circledR}$ (Ethicon Endo-Surgery, Johnson \& Johnson, Germany) monopolar diathermy device we use is equipped with an inbuilt suction mechanism which is very useful during intraoperative bleeding (Figure 6). Authors also use 'tonsillar swabs' to apply pressure and use the same to apply upward traction on the rectum. One specific site of troublesome bleeding is the middle rectal artery, which can be well defined, especially on the left side and would require clipping before division. Recognition of opening in to deeper lateral planes is helped by the 'Halo' sign described by Bernardi et al [6]. They described the appearance of an 'O' shape defect when a new fascial plane is opened. However, this is not an absolute sign but should alert the operator to re-evaluate. Another well recognized challenge during dissection is the anterior prostatic plane in UC patients [6]. This area anyway poses a challenge due to the close proximity of the urethra and prostate to the rectum, although in this particular group of patients the plane was heavily fibrotic. This is an interesting observation, as UC is not expected to cause trans-mural inflammation. Concurrent abdominal dissection is helpful in guiding the two dissection planes to meet each other. Results from TaTME in benign disease is lacking in comparison to its use in malignant disease.

A recent report by de Buck van Overstraeten demonstrated a comparable complication rate and lesser postoperative morbidity in TaTME with IPAA compared to conventional laparoscopic pouch surgery [7]. Comparing 97 TaTME procedures with 119 trans-abdominal procedures, they reported a significantly lower complication rate and a lower comprehensive complication index (CCI) in the TaTME group. Although IBD is a benign disease, the authors tend to carry out dissection in the TME plane for several reasons. The current patient cohort had an incidental cancer detection rate of $7 \%$ in the dissected specimens. The field change in the colorectal mucosa that occurs with inflammation, predisposes to dysplasia and cancer that may go undetected in focused biopsies. Carrying out a close dissection technique could compromise the oncological safety in this group of patients. Also, a yet unproven theory suggests the perirectal or peri colic fat in Crohn's disease contributes to the inflammation. Lindsey and colleagues have observed no difference in the rates of sexual dysfunction following either technique in IBD [8]. Therefore, the authors feel that the argument for a close rectal dissection lacks support. Patients with perineal Crohn's who undergo completion proctectomy, pose a challenges in the management of the perineal wound. Defects following excision in infected fields are managed with vacuum dressings followed by a flap cover (Figure 5). Surprisingly, some of these large defects contract and granulate significantly, not requiring a flap cover (Figure 6). Uncomplicated wounds in the perineum are closed in layers vertically with an abdominal drain placed in the pelvis.

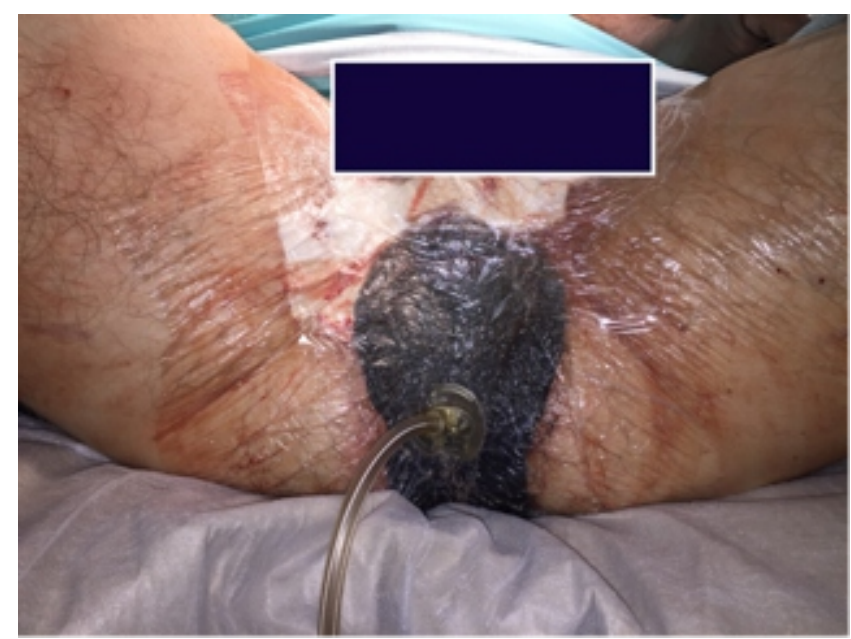

Figure 5. Vacuum dressing applied to a perineal defect in a ptient with Crohn's disease following TaTME.

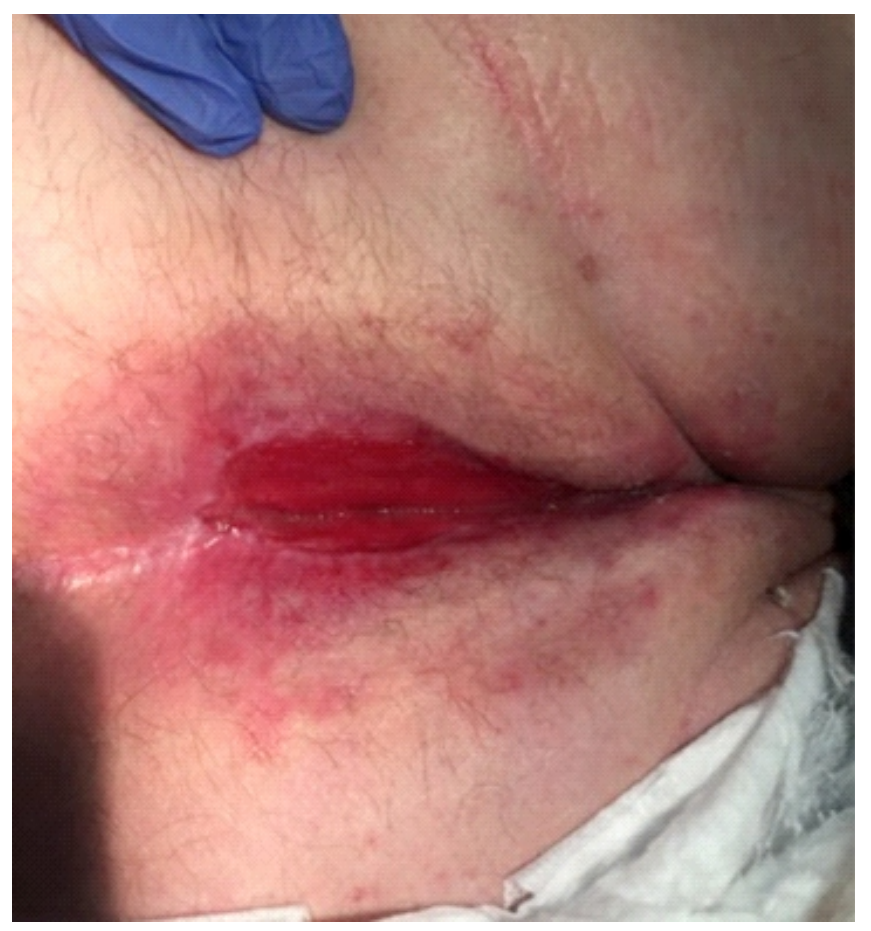

Figure 6. Wound constricted 6 weeks after the application of vacuum dressing. 
A common complication to look out for in the immediate postoperative period is wound breakdown, although caution in decision-making as to which wounds can be closed primarily could prevent this. Some of these wounds which fail, might result in a chronic discharging sinus [9]. Another complication related to completion proctectomy in $\mathrm{CD}$ patients, is a pelvic collection [10]. These collections may be managed conservatively or with intervention in the form of radiological guided aspiration, depending on the size and the sinuses with the use of video assisted anal fistula treatment (VAAFT) technique. Accumulation of more outcome data on this relatively novel technique, will allow the surgeons to evaluate its advantages and disadvantages over conventional trans-abdominal surgery.

\section{Conclusion}

In this article we describe the techniques and challenges related to transanal resection for inflammatory bowel disease. We have demonstrated that TaTME is technically feasible in IBD and offers some advantages over standard laparoscopy, especially in relation to allowing control of the point of rectotomy and anastomosis. Post-operative wound management following trans anal surgery in this cohort of patients is at times challenging, although the results may be favourable in the long term.

All authors disclose no conflict of interest. The study was conducted in accordance with the ethical standards of the relevant institutional or national ethics committee and the Helsinki Declaration of 1975, as revised in 2000 .

\section{References}

1. Buess G, Theiss R, Gunther M, Hutterer F, Pichlmaier H. Endoscopic surgery in the rectum. Endoscopy. 1985;17(1):31-5. https://doi.org/10.1055/s-2007-1018451

2. Atallah S, Albert M, Larach S. Transanal minimally invasive surgery: A giant leap forward. Surg Endosc Other Interv Tech. 2010;24(9):2200-5. https://doi.org/10.1007/s00464-010-0927-z

3. Velthuis S, van den Boezem PB, van der Peet DL, Cuesta M a, Sietses C. Feasibility study of transanal total mesorectal excision. Br J Surg. 2013;100(6):828-31; discussion 831 . https://doi.org/10.1002/bjs.9069

4. Dumont F, Goéré D, Honoré C, Elias D. Transanal Endoscopic Total Mesorectal Excision Combined With Single-Port Laparoscopy. Dis Colon Rectum. 2012;55(9):996-1001. https://doi.org/10.1097/DCR.0b013e318260d3a0

5. Lacy AM, Adelsdorfer C, Delgado S, Sylla P, Rattner DW. Minilaparoscopy-assisted transrectal low anterior resection (LAR): a preliminary study. Surg Endosc. 2013 Jan 18;27(1):339-46. https://doi.org/10.1007/s00464-012-2443-9

6. Bernardi M-P, Stevenson ARL, Hompes @bullet R. Transanal total mesorectal excision: dissection tips using "O"s and "triangles." Tech Coloproctol. 20.

7. Buck van Overstraeten A, Mark-Christensen A, Wasmann KA, Bastiaenen VP, Buskens CJ, Wolthuis AM, et al. Transanal Versus Transabdominal Minimally Invasive (Completion) Proctectomy With Ileal Pouch-anal Anastomosis in Ulcerative Colitis. Ann Surg. 2017 Nov;266(5):878-83. https://doi.org/10.1097/SLA.0000000000002395

8. Lindsey I, George BD, Kettlewell MGW, Mc. C. Mortensen NJ. Impotence after mesorectal and close rectal dissection for inflammatory bowel disease. Dis Colon Rectum. SpringerVerlag; 2001 Jun;44(6):831-5.

9. Maeda Y, Heyckendorff-Diebold T, Tei TM, Lundby L, Buntzen S. Gracilis muscle transposition for complex fistula and persistent nonhealing sinus in perianal Crohn's disease. Inflamm Bowel Dis. 2011 Feb;17(2):583-9. https://doi.org/10.1002/ibd.21311

10. Yamamoto T, Bain IM, Allan RN, Keighley MRB. Persistent perineal sinus after proctocolectomy for Crohn's disease. Dis Colon Rectum. Springer-Verlag; 1999 Jan;42(1):96-101. 